FZMw Jg. 1 (1998) H. 1, S. 40-50

\title{
Edvard Grieg als Musikerzieher - Eine Anmerkung
}

\author{
von Klaus Henning Oelmann
}

Im Jahrbuch 1997 der Reihe Musikpädagogische Forschung, das sich der musikpädagogischen Biographieforschung widmet, veröffentlichte Hella Brock eine Betrachtung zu Edvard Grieg als Musikerzieher [1]. Sie macht darin der "musikhistorischen Biographik" zu Recht den Vorwurf, daß eine Reihe von Fragen, die im Zusammenhang mit der pädagogischen Tätigkeit berühmter Komponisten entstehen, "nur peripher beantwortet wurden". Zu diesen rechnet sie

$\circ$ das Verhältnis von "nationalen und gesellschaftlich-kulturellen Entwicklungsprozesse[n]" sowie des Komponisten "Erfahrungen und Persönlichkeitseigenschaften" und seine pädagogischen Intentionen,

○ den Stellenwert, den diese Intentionen im Laufe seiner Karriere erlangen,

○ die Arbeitsbereiche, in denen sie sich entfalten können, und

○ die Kennzeichnung bestimmter Werke als pädagogisch konzipierte nebst deren Verhältnis zum "künstlerisch-ästhetischen Anspruch".

Es versteht sich, daß diese Fragen bei einem Komponisten, dessen Euvre in Deutschland einer durchaus zwiespältigen Würdigung unterliegt, von besonderem Interesse sind. Im folgenden soll versucht werden, aus musikwissenschaftlicher und historischer Sicht auf einzelne Aspekte dieses umfangreichen Fragenkomplexes einzugehen.

Die Bemühungen, ein Persönlichkeitsbild von Edvard Grieg zu zeichnen, haben in der in Deutschland zugänglichen Literatur eine lange Tradition. Daß man sie als fragwürdig bezeichnen kann, liegt daran, daß sie in der Regel von zwei Veröffentlichungen ausgeht, deren Quellenwert heute bestritten werden muß. Es handelt sich einmal um Griegs autobiographische Skizze Mein erster Erfolg, 1905 fast zeitgleich in Velhagen \& 
Klasings

SEITE: 40

Monatsheften (S. 531-540) und im 27. Heft der Neuen Musikzeitung abgedruckt, und um Arthur Abells Interviewsammlung Talks with Great Composers (New York 1950), in deutscher Übersetzung mit dem Untertitel So entstanden ihre unsterblichen Meisterwerke 1962 in Eschwege und Garmisch-Partenkirchen erschienen.

Bis in die 90er Jahre hinein sind diese Schriften nahezu kritiklos rezipiert worden [2], obwohl Joachim Reisaus' von Hella Brock betreute Dissertation 1988 nachwies, daß Griegs eigene Einlassungen gewisse Filter passiert hatte, die sie als Primärquelle unbrauchbar machen [3]. Abells Interviews haben, da sie neuerer Forschung diametral entgegenstehen, sogar Zweifel geweckt, ob sie in der publizierten Form jemals zustandegekommen sind [4]. Bei der auch von Brock gerühmten Fülle an anderen zugänglichen Selbstzeugnissen ist das Festhalten an derartigem Material unverständlich.

Brock wählt in ihrem Versuch der Persönlichkeitsbeschreibung Griegs den Weg über die neuere norwegische Geschichte, belegt aber ihre diesbezüglichen Thesen nur mangelhaft oder gar nicht. Es stört darüber hinaus ein Tonfall, der wissenschaftstheoretisch überholt erscheint. Ein "bürgerlich-revolutionäres Denken" (S. 53) hat es in Norwegen in Zusammenhang mit der Loslösung von Dänemark in der Tat nicht gegeben, die Französische Revolution hatte fürs erste so gut wie keinen Widerhall in Norwegen gefunden. Ein Blick in die einschlägige Literatur weist aus, daß die nationale Bewegung um die Jahrhundertwende in ihrer Bedeutung eher nachließ. Die Neutralität Dänemarks während der kontinentalen Kriege des 18. Jahrhunderts ließ die Bedeutung der norwegischen Handelsflotte stark anwachsen; auch hatte Dänemark erfolgreich versucht, die Bindungen beider Reichsteile zu stärken, indem etwa dem 
Kernland mit wenigen Ausnahmen das Kornhandelsmonopol zustand, im Gegenzug Norwegen das Eisenwarenhandelsmonopol

eingeräumt wurde. Bis zum Jahre 1807, als England im Zuge der Napoleonischen Kriege den Kornhandel auf See unterband, ließ die ökonomische Prosperität die nationale Frage eher in den Hintergrund treten.

Der "dänische Feudalabsolutismus", den Brock beschreibt (S. 53), wurde bereits um 1784 gelockert, als in mehreren Schritten sowohl die Freiheit des gedruckten Wortes eingeführt als auch die Leibeigenschaft der Bauern aufgehoben wurde.

Das, was Brock "die erste freigewählte norwegische Nationalversammlung" nennt, war eine durch indirekte Wahl zustandegekommene Delegiertenkonferenz, die über die Frage der Selbständigkeit befinden und ein Grundgesetz ausarbeiten sollte, und überdies auf Initiative des dänischen Statthalters in Norwegen, Prinz Kristian Fredrik, zustandegekommen war. Es ist womöglich richtig, daß die am 17. Mai 1814 verabschiedete Verfassung "wesentlich demokratischer war als jede andere in Europa" (S. 53), indem sie etwa die Macht zwischen König und Reichstag teilte, ein Wahlrecht konstituierte, welches einen relativ großen Teil der Bevölkerung umfaßte, und die allgemeine Druckfreiheit sicherte. Gern wird dabei vergessen, daß die Verfassung u. a. auch einen Artikel enthielt, der den Zuzug von Juden nach Norwegen verbot, und die nationalen Minderheiten der Samen und Kvenen nicht miteinbezog. Und daß ein Grund für die liberale Ausrichtung der Verfassung gewesen wäre, daß "sich [...] das Bürgertum [...] auf eine Bauernschaft stützen" konnte, "die von jeher die freiheitlichen Traditionen des norwegischen Volkes verkörperte" (S. 53), stimmt so nicht. Schnell erwies sich nämlich, daß die faktisch vorhandene tiefe Kluft zwischen Bauern und Städtern zu 
einem Kulturkampf ohnegleichen führte, der bis heute seine Wellen schlägt. In der Frage der zu entwickelnden Staatssprache - das alte Schriftnorwegisch war um 1525 ausgestorben - standen sich die Befürworter einer allmählichen Ablösung vom Dänischen, in der Regel Bewohner der dichter besiedelten süd- und mittelnorwegischen Küstenregionen, und die Anhänger einer radikalen Erneuerung der Sprache auf der Grundlage der ländlichen Dialekte unversöhnlich gegenüber, und bis heute leistet sich Norwegen den Luxus zweier Staatssprachen, deren Entfaltung von staatlicher Seite streng überwacht ist und von den jeweiligen Parteigängern mit Argusaugen verfolgt wird. Insofern kann von einem einheitlichen "progressive[n] Bürgertum" (S. 54) im 19. Jahrhundert nicht die Rede sein. In dem Maße, in dem sich Künstler und Intellektuelle in dem Streit engagierten, verschwand auf kultureller Ebene, aber eben nur dort, die Bedeutung der Klassenschranken [5].

Grieg - ein wesentliches Element in des Komponisten eigenem nationalen Verständnis ergriff zunächst vehement Partei für die Vertreter der 'Bauernsprache' (landsmaal, heute nynorsk) und setzte ihr mit den Liederzyklen op. 33 und 67 ein musikalisches Denkmal. Die zunehmende Polemik auf beiden Seiten beobachtete er jedoch mit Skepsis und untersagte schließlich dem Dichter Arne Garborg die Veröffentlichung ihres Briefwechsels, mit dem dieser auf die überzogenen Anwürfe, etwa von Bjørnstjerne Bjørnson, reagieren wollte [6].

$$
-8-
$$

Brocks Aufzählungen von Persönlichkeiten im Umkreise Griegs, die ihn ermutigten (S. 55) und ihn seine "Lebensaufgabe als Komponist, Interpret und Musikerzieher" (S. 54) erkennen lassen sollten, sagen schon insofern wenig aus, als sie unter allgemeine Werte wie nationale Unabhängigkeit und Demokratie gestellt werden. Auch existiert bislang 
keinerlei Beleg dafür, daß Grieg die von Brock intendierte Dreiwertigkeit dieser Lebensaufgabe jemals so gesehen hat. Im Gegenteil gibt es in der Korrespondenz Griegs Hinweise darauf, daß dieser die pädagogische und konzertierende Arbeit eher als Last empfand, die ihn vom Komponieren abhielt. Seine Arbeit als Klavierlehrer in Oslo muß im Lichte seiner Bemühungen gesehen werden, seiner Familie überhaupt ein regelmäßiges Einkommen zu verschaffen [7]. Ähnlich verhält es sich mit seinem Engagement als Dirigent der Musikgesellschaft Harmonien in Bergen, das erst nach mehrmaligem Insistieren der Intendanz und nicht ohne erhebliche Aufbesserung des Salairs zustandekam. Bereits nach zwei Saisons gab Grieg den Stab weiter und zog sich zur Kur nach Karlsbad zurück [8].

\section{- 9 -}

Mit Zurückhaltung müssen sodann die Zitate betrachtet werden, die Hella Brock zur Stützung ihrer These von der Gegnerschaft Griegs hinsichtlich einer "Exklusivität der Musik" gebraucht (S. 54f). Zunächst einmal war Grieg 1899 längst auf dem Weg zu einem Status als Modekomponist, wie es Helmut Link, seinerzeit Dirigent des HaydnOrchesters Berlin, auszudrücken pflegte. Seine Werke hatten sowohl die Konzertsäle als

auch die Stuben der Amateurpianisten erobert; Max Abraham, Griegs Verleger und freundschaftlicher Berater, bezeichnete die erste Suite aus der Schauspielmusik zu Peer Gynt op. 46 aus dem Jahre 1888 vier Jahre später als "unbedingt eins der allerpopulärsten Orchesterwerke der Neuzeit" [9]. Insofern ist der von Brock beschriebene Erfolg Griegs im Arbeiterverein zu København im besagten Jahr erfreulich, aber nicht allzu verwunderlich.

Zum anderen ist zu bedenken, daß Griegs von Brock zitierte Tirade auf "Dieses verdammte blasirte und glasirte Publikum" (S. 55) wieder einmal Leipzig und die 
Københavner Musikforening umfaßte: Im ersten Falle machte ihm die verständnis- und kenntnislose Journaille Erich Bernsdorfs zu schaffen, im zweiten sah er seit seinen Auseinandersetzungen mit Niels Wilhelm Gade in den 60er Jahren prinzipiell den Rückschritt am Werke [10]. Nur am Rande sei bemerkt, daß Leipzig 1890 selbst einem für Nichtnorweger schwer verständlichen Werk wie dem Opernfragment Olav Trygvason op. 50 zugejubelt hatte, Griegs Vorwürfe von daher der Grundlage entbehren.

$$
-11-
$$

Die " Mittel der fortgeschrittensten Kompositionspraxis seiner Zeit" (S. 56), denen Brock im Zusammenhang mit Griegs Bearbeitungen norwegischer Volksweisen das Wort redet, verdienten eine eingehendere Betrachtung, als sie hier geleistet werden kann. An anderer Stelle ist schon auf das nach Meinung des Verfassers falsche Verständnis einer analytischen Betrachtungsweise von Musik hingewiesen worden, die sich den zugrundegelegten analytischen Kriterien entzieht [11]. Kompositionen, die sich ausdrücklich und nachprüfbar auf ihre volksmusikalischen Wurzeln berufen, wird man kaum mit einem Analyseapparat beikommen, der die musikalischen Phänomene der Vorlage nicht in entsprechende Kategorien fassen kann. So müßte zum Beispiel im vorliegenden Falle - Griegs in einem Brief an seinen amerikanischen Biographen Henry T. Finck geäußerte Ansicht von den "verborgenen Harmonien" (S. 56) der Volksmusik beim Wort nehmend - erst einmal geklärt werden, inwieweit ostinate Spielweisen und Bordunstimmung der hardingfela (Hardangerfidel) bereits Bedingungen für die entstehenden Harmonien darstellen. Indem man Grieg den Fortschritt aufgrund einer bestimmten Analysepraxis einfach konstatiert, unterläuft man gewollt oder ungewollt alle Möglichkeiten, das Besonde-

re, konservative Hörgewohnheiten Reizende an Griegs Musik darzustellen, wie Friedhelm Krummacher es eingefordert hat [12]. 
Brocks Ansicht, die Lyrischen Stücke dienten in erster Linie dazu, den Spieler "Naturerlebnisse und die Sagenwelt, die Träume und die Sehnsüchte des norwegischen Volkes in vielfältigen Klängen der Heimat nachempfinden" (S. 57) zu lassen, steht die Tatsache entgegen, daß Grieg gerade in die zehn Hefte dieser Gattung nicht eine einzige volksmusikalische Vorlage aufnahm. Brock erleichtert dem heutigen Rezipienten damit ein Vorurteil, mit dem schon Grieg zu kämpfen hatte, nämlich daß er in der Musik doch nichts weiter als einen norwegischen Dialekt spräche. Nicht erst seit dem verdienstvollen Essay Harry Goldschmidts sollte jedoch klar sein, daß Grieg "Kunst im Weltmaßstab" [13] wollte. Auch scheint Brock von den Untersuchungen Frank Dörschels (op.cit.) keine Kenntnis zu haben, der gerade versuchte, das Spannungsfeld der Kompositionen "zwischen norwegischer Nationalkunst und deutscher Tradition" so der Titel seiner Untersuchung - auszuloten.

$$
-13-
$$

Brocks Argumentation hinsichtlich der Absage von Griegs Konzerten in Paris 1899 und in St. Petersburg 1904 (S. 59) wirkt nicht schlüssig. Es ist richtig, daß Grieg diesbezügliche Einladungen wegen der wieder aufgeflammten Dreyfus Affaire und des Japanisch-Russischen Krieges abgelehnt hatte. Doch wäre etwas an der "hohe[n] ethische[n] Funktion" (S. 59), die Brocks Ansicht nach Grieg der Musik zuwies, hätte ihn diese doch bewegen müssen, nun erst recht dort zu konzertieren, wo diese Funktion womöglich etwas hätte ausrichten können. Auch in den zunehmenden Spannungen zwischen Norwegen und Schweden hatte Grieg 1896 ja seinen eigenen Worten nach "eine nationale Mission" erfüllt, indem er dazu beigetragen habe, "das schwedische und norwegische Volk einander näher zu bringen" (S. 59). Es kann jedoch als fraglich gelten, ob sich Grieg der langfristigen Wirkungen seiner politischen Einlassungen überhaupt bewußt war. Im Gegensatz zu Bjørnstjerne Bjørnson, der seinen Einfluß als Intellektueller und nicht zuletzt als Redner im Aufkeimen der nationalen Frage in der habsburgischen Monarchie kalkulierte und langfristig einsetzte [14], gab Grieg in der Regel Augenblicksstimmungen nach, deren Weiter- und Nachwirkungen er nicht bedachte. So konzertierte er Jahre später 
doch in Paris - die Sache Dreyfus war mitnichten ausgestanden - und war ernsthaft überrascht über die feindliche Haltung, die ihm ein Teil des Publikums entgegenbrachte [15].

Brocks Kriterien, die sie zur Beschreibung von für Kinder spielbaren volksmusikalischen Bearbeitungen Griegs entwickelt, wecken Befremden. Der Satz "Die meisten von ihnen sind zugleich für erwachsene Klavierspieler interessant, zumal Grieg gerade diesen Kinderliedweisen bei ihrer Bearbeitung besondere Sorgfalt zugewandt hat" (S. 60) legt nahe, er habe es bei anderen Bearbeitungen an dieser Sorgfalt fehlen lassen. Das ist nicht der Fall, wie man beispielsweise beim Blick in die Partitur der 19 norwegischen Volksweisen op. 66 unschwer feststellen kann. Und daß in der Sequenzierung einer pentatonisch aufsteigenden Tonfolge etwa "die Zärtlichkeit gegenüber dem müden Pferdchen unmittelbar zum Ausdruck" (S. 61) gebracht wird, ist einem aufmerksamen Zuhörer kaum zu vermitteln. Ein solcher wird es vermutlich ablehnen, seiner eigenen Phantasie derart die Zügel anlegen zu lassen.

$\mathrm{Zu}$ den Kinderliedern (eigentlich genauer: Kindlichen Liedern) op. 61 schreibt Brock: "Grieg war um die Vertonung von Gedichten gebeten worden, die für ein Lesebuch zusammengestellt worden waren. Er erfüllte diesen Auftrag mit großer Freude" (S. 61). In Griegs erster Erwähnung der Lieder gegenüber Max Abraham ist von Freude gerade nicht die Rede: "Nach Aufforderung habe ich für die Schule verschiedene Lieder machen müssen", heißt es am 29. Juli 1894. Am 21. Dezember schränkt er weiter ein: "Einige daraus gefallen mir", und klagt am 10. Juni 1895:

"Die sogenannten Kinderlieder hätte ich übrigens nicht so leichtsinnig behandeln sollen. Auch Nichts von dem was ich je componirte, hat eine derartige Popularitet erlangt, nicht nur in der Heimat, sondern auch in Koncerten. Schauderhaftes Pech !"

Dazu paßt, daß Grieg nicht daran dachte, die Lieder außerhalb Norwegens zu 
veröffentlichen, und damit für nicht geringe Aufregung bei Peters sorgte: Seit 1889 in Generalkontrakt mit Grieg stehend, sah man sich unversehens mit dem Druckvorhaben eines Konkurrenten konfrontiert, der die Rechte für op. 61 aus Norwegen erworben hatte [16].

Brocks Ausführungen zur Entstehung und Rezeption der Slåtter (Norwegischen Bauerntänze) op. 72 ist im allgemeinen zuzustimmen. 1901 hatte Grieg seinen Landsmann Johan Halvorsen beauftragt, Tänze aus dem Repertoire des Spielmannes Knut Dahle aufzuzeichnen. Im Sommer 1902 bearbeitete er 17 von diesen für Klavier solo und setzte weitsichtig die gleichzeitige Veröffentlichung von Transkription und Arrangement bei Peters durch:

"Die Arbeit Halvorsens ist [...] eine treue, und zwar vollendete Aufzeichnung der Bauerntänze, so wie die Fiedler dieselben ausführen. Er selbst hat keinen Ton hinzugefügt. Da es das erste Mal ist, wo solche norwegischen Originaltänze der Öffentlichkeit vorgelegt werden, ist gerade dieser Umstand [...] für den Forscher von der höchsten Interesse und Sie haben dadurch - vielleicht ohne es zu wissen - sowohl die Musikwissenschaft wie Norwegen einen nicht geringen Dienst gethan."

schrieb er am 4. September 1903 an Peters. Ob es allerdings nach Brock nur die intrikate Setzweise der Bearbeitungen [17] war, die die zunächst zögerliche Aufnahme im Heimatland erklärte (S. 63), bedarf angesichts von Griegs Gesundheitszustand der Differenzierung. Seit der Jahrhundertwende hatte Grieg zu kränkeln begonnen; eine alte Instabilität der Luftwege, von einer Lungenerkrankung in der Kindheit herrührend, machte sich mehr und mehr geltend. Einige größere Projekte, u. a. ein drittes Streichquartett und ein Klavierquintett, hatte Grieg aufgeben müssen, und womöglich hatten auch seine Fähigkeiten als Pianist gelitten. Bei Peters jedenfalls mochte er keinen 
der Tänze vorführen [18], und vielleicht hat es auch mit einer nicht recht geratenen Aufführung Griegs zu tun, daß man sich in seiner Heimat abwartend verhielt. Erst gegen Ende seines Lebens fand Grieg in Percy Grainger den kongenialen Interpreten seiner volksmusikbasierten Klavierkompositionen.

$$
-17 \text { - }
$$

"Griegs Intentionen als Musikerzieher duldeten keine Abstriche von seiner Arbeit als Komponist", folgert Hella Brock (S. 63). Warum aber dann ein solcher Artikel? Ein wirklich erzieherisches Interesse Griegs, das über allgemeine Absichtserklärungen hin-

ausginge, kann Brock an keiner Stelle belegen. In der Komposition des Op. 72 wird zudem eher ein starkes kulturhistorisches denn ein pädagogisches Interesse Griegs sichtbar. Eine ganz andere Sache ist die Verwendung der Tänze im Musikunterricht, die Brock am Schluß ihres Beitrags andeutet; diese mag in der entsprechenden didaktischen Aufbereitung möglich sein, kann jedoch kaum für ein pädagogisches Konzept Griegs einstehen.

Es gibt andererseits ein Werk, das tatsächlich geeignet sein könnte, musikpädagogische Thesen an Grieg zu erproben, und das bei Brock nicht einmal erwähnt wird, die 154 auf Griegs Geheiß zunächst anonym veröffentlichten Lieder in Norges melodier (Norwegens Melodien). Hier hatte Grieg ebenfalls auf Anfrage teils eigene Stücke, teils die anderer Komponisten und teils Volkslieder für den allgemeinen Gebrauch bearbeitet. $\mathrm{Zu}$ zeigen, wie Grieg auswählte, und wie er die Auswahl dem Bedarf anpaßte, sollte doch eine Fundgrube für historisch-musikpädagogische Forschung sein.

$$
-19-
$$

Daß Brock ihre Untersuchungen fast ausschließlich auf eigene Quellen stützt, trägt nicht 
dazu bei, die Vielschichtigkeit Griegs als Person und Komponist auszuloten [19]. Es ist hier versucht worden, die Einseitigkeit von Brocks Betrachtungen durch zahlreiche Verweise aufzubrechen. In der zitierten Literatur wird man weitere Hinweise finden, die das Bild des norwegischen Komponisten weiter auffächern können.

$$
-20-
$$

Die Frage schließlich, ob Brocks Artikel ihren Kriterien zur Erforschung der musikpädagogischen Wirksamkeit großer Komponisten gerecht wird, muß verneinend beantwortet werden. Die nationalen und gesellschaftlich-kulturellen Entwicklungsprozesse zur Zeit Griegs sieht Brock - bei allen Diskussionsmöglichkeiten jedweder geschichtswissenschaftlicher Thesen - in falschem Licht, Griegs pädagogische Intentionen werden unrealistisch überhöht. Werke, die diese Intentionen belegen könnten, werden nicht behandelt, hingegen andere Kompositionen herangezogen, die eher außerhalb des gegebenen Zusammenhangs stehen. Griegs Wirkungsfelder werden ausführlich beschrieben, ihre Wurzelung im Pädagogischen bleibt jedoch als ungenügend belegte These stehen. Durch die Auslassung tatsächlich pädagogisch relevanter Werke wird eine Chance vertan, die überaus wichtige Fragestellung der Verankerung künstlerisch-ästhetischen Anspruches in

musikpädagogisch ausgerichteten Werken im Vergleich zu anderen Komponisten zu beleuchten und zu vertiefen.

Literatur- und Quellenverzeichnis:

Benestad, F. \& Schjelderup-Ebbe, D., Edvard Grieg - Der Mensch und der Künstler, Leipzig, Deutscher Verlag für Musik, 1993

Blok, Vladimir, Grieg - Bartók, Grieg - Prokofiev: Aesthetic Parallels, in: Studia musicologica norvegica 19, 1993, S. 239-250 
Bull, Edvard, Nordmenn før oss, Oslo: Tanum Nordli, ${ }^{6} 1976$

Brock, Hella, Grieg und Deutschland, in: Studia, 1993, S. 97-102

Dies., Edvard Grieg als Musikerzieher, in: Musikpädagogische Biographieforschung: Fachgeschichte - Zeitgeschichte - Lebensgeschichte (Musikpädagogische Forschung Bd. 18, hrsg. von Rudolf-Dieter Kraemer), Essen: Die Blaue Eule, 1997, S. 52-63

Dörschel, Frank, Griegs 'Lyriske stykker' zwischen norwegischer Nationalkunst und deutscher Tradition, M.A. Frankfurt/Main, 1995

Donnellon, Deirdre, Grieg, Debussy and the Dreyfus Affair, in: British Postgraduate Musicology 1, 1997, S. 14-19

Goldschmidt, Harry, Um die Sache der Musik, Leipzig: Reclam, 1970

Grieg, Edvard, Briefwechsel Bd. 2: Der Briefwechsel mit dem Hause Breitkopf \& Härtel [e.a.], hrsg. von Klaus H. Oelmann (Deutsche Hochschulschriften Bd. 1123), Egelsbach / St. Peter Port: Hänsel-Hohenhausen / Knaresborough Ltd., 1996

Ders., Briefwechsel mit dem Musikverlag C.F. Peters 1863-1907, hrsg. von Finn Benestad und Hella Brock, Frankfurt/Main: Peters, 1997

Herresthal, Harald, Artikel Norwegen und Oslo, in: Die Musik in Geschichte und Gegenwart, 2., neubearb. Ausgabe, hrsg. von Ludwig Finscher, Band 7, 1997, Sp. $250-261 / 270-272 / 1155-1163$

Kortsen, Bjarne, Norwegian Music and Musicians, Bergen: Edition Norvegica, ${ }^{2} 1977$

Ders., Grieg the Writer - Le style c'est l'homme même, in: Christensen, Mogens e.a. (Hrsg.): Die Gratulanten kommen Edvard Grieg zum 150. Geburtstag (Deutsche Hochschulschriften Bd. 814), Egelsbach: Hänsel-Hohenhausen, 1993, S. 58-72

Krummacher, Friedhelm, Rezeptionsgeschichte in der Musikwissenschaft Möglichkeiten und Grenzen im Rückblick, in: Kongreßbericht Oslo (Skriftserie fra Institutt for musikk og teater ved Universitetet i Oslo), 1992, S. 213-230

Noreng, Harald, Der Sprachkünstler und Literaturliebhaber Edvard Grieg, in: Christensen (Hrsg.): Die Gratulanten, 1993, S. 77-87 
Oelmann, Klaus H., Edvard Grieg - Versuch einer Orientierung (Deutsche Hochschulschriften Bd. 485), Egelsbach: Hänsel-Hohenhausen, 1993

Reisaus, Joachim, Grieg und das Leipziger Konservatorium - Untersuchungen zur Persönlichkeit des norwegischen Komponisten unter besonderer Berücksichtigung seiner Leipziger Studienjahre, Diss. Leipzig, 1988

SEITE: 49

Schjelderup-Ebbe, Dag, Edvard Grieg 1858-1867, Oslo / London: Universitetsforlaget / Allan \& Unwin, 1964

Andere Literatur zur volksmusikbasierten Klaviermusik Griegs:

Kleiberg, Ståle, Grieg's Slåtter, Op. 72 - Change of Musical Style or New Concept of Nationalism?, in: Journal of the Royal Musical Association 1, 1996,:S. 46-57

Ledang, Ola K., Individual Creation and National Identity - On Grieg's Piano Adaptations of Hardingfele Music, in: Studia, 1993, S. 39-44

Sevåg, Reidar, Tanzmusik auf der Hardangerfidel - Eine hochentwickelte solistische Spielpraxis, in: Stockmann, Doris (Hrsg.): Volks- und Popularmusik in Europa (Neues Handbuch der Musikwissenschaft Bd. 12), Laaber: Laaber Verlag, 1992

\section{Anmerkungen:}


1 Brock, Hella, Edvard Grieg als Musikerzieher, in: Musikpädagogische Biographieforschung: Fachgeschichte - Zeitgeschichte - Lebensgeschichte. (Musikpädagogische Forschung Bd. 18, hrsg. von Rudolf-Dieter Kraemer), Essen: Die Blaue Eule, 1997, S. 52-63, im folgenden zitiert mit der entsprechenden Seitenzahl. Die im ersten Absatz gekennzeichneten Zitate stehen auf S. 52.

\section{[Zurück zum Text]}

${ }^{2}$ Vgl. etwa die entsprechenden Abschnitte in Benestad, F. \& Schjelderup-Ebbe, D., Edvard Grieg - Der Mensch und der Künstler, Leipzig: Deutscher Verlag für Musik, 1993. Auch die 1997 erschienene Ausgabe von Griegs Korrespondenz mit dem Petersverlag zitiert unvorbehalten aus Abells Buch und rühmt Mein erster Erfolg als "stilistisches Meisterwerk, das dem Leser die Kindheitserlebnisse Griegs, seine Freuden und Enttäuschungen während der Konservatoriumszeit in Leipzig in lebendigen Bildern vermittelt", immerhin mit dem Zusatz, daß "einige seiner Einschätzungen von subjektiven Übertreibungen gewiß nicht frei sein mögen." (Grieg, Edvard, Briefwechsel mit dem Musikverlag C.F. Peters 1863-1907, hrsg. von Finn Benestad und Hella Brock, Frankfurt/Main: Peters, 1997, S. 467 u. 516).

\section{[Zurück zum Text]}

${ }^{3}$ Reisaus, Joachim, Grieg und das Leipziger Konservatorium - Untersuchungen zur Persönlichkeit des norwegischen Komponisten unter besonderer Berücksichtigung seiner Leipziger Studienjahre, Diss. Leipzig, 1988. Vgl. auch die Diskussion der Reisausschen Thesen in Oelmann, Klaus H., Edvard Grieg Versuch einer Orientierung (Deutsche Hochschulschriften Bd. 485), Egelsbach: Hänsel-Hohenhausen, 1993, S. 46 u. 160ff., und Dörschel, Frank, Griegs 'Lyriske stykker' zwischen norwegischer Nationalkunst und deutscher Tradition, M.A. Frankfurt/Main, 1995, S. 45. Reisaus' Versuch einer postmortalen Anamnese Griegs ist seinerseits nicht unproblematisch, was eine eigene, psychologisch ausgerichtete Untersuchung wert wäre.

\section{[Zurück zum Text]}

${ }^{4}$ Vgl. Oelmann, Versuch, S. 161f, und Dörschel, Lyriske stykker, S. 148, 152.

\section{[Zurück zum Text]}

5 Vgl. zu den vorigen Abschnitten Bull, Edvard, Nordmenn før oss, Oslo: Tanum Nordli, ${ }^{6} 1976$, S. 161-175, 192-197.

\section{[Zurück zum Text]}

${ }^{6}$ Vgl. Oelmann, Versuch,. S. 119-125, 135f. Vor diesem Hintergrund ist Griegs "heiße[s] Herz" (S. 54) gerade auf dem Gebiet der kulturellen Selbstbestimmung Norwegens Wunschdenken.

\section{[Zurück zum Text]}

${ }^{7}$ Was Hella Brock auch selbst einräumt (S. 57, 63).

[Zurück zum Text]

${ }^{8}$ Siehe z. B. den Peters Briefwechsel, S. 136, 316, 321; auch Grieg, Edvard, Briefwechsel Bd. 2: Der Briefwechsel mit dem Hause Breitkopf \& Härtel, [e.a.] (Deutsche Hochschulschriften Bd. 1123) Egelsbach / St. Peter Port: Hänsel-Hohenhausen / Knaresborough Ltd., 1996, S. 41, 43, 51, 53. Was den bei Brock erwähnten Rikard Nordraak betrifft, war er von derart exzentrischer Persönlichkeit, daß man ihm ein ernsthaftes, über seine eigene Person hinausreichendes Engagement für nationale Unabhängigkeit und Demokratie kaum zutrauen mochte; vgl. Schjelderup-Ebbe, Dag, Edvard Grieg 1858-1867, Oslo / London, Universitetsforlaget / Allan \& Unwin, 1964, S. 216-281. 


\section{[Zurück zum Text]}

${ }^{9}$ Peters Briefwechsel, S. 270. Der entsprechende Hinweis fehlt im Register.

\section{[Zurück zum Text]}

10 Siehe den Breitkopf Briefwechsel, S. 62f, und die entsprechenden Kapitel in Benestad \& Schjelderup-Ebbe, Der Mensch, vgl. auch Krummacher, Friedhelm, Rezeptionsgeschichte in der Musikwissenschaft - Möglichkeiten und Grenzen im Rückblick, in: Kongreßbericht Oslo (Skriftserie fra Institutt for musikk og teater ved Universitetet i Oslo), 1992, S. 213-230.

\section{[Zurück zum Text]}

11 Oelmann, Versuch, S. 18ff.

[Zurück zum Text]

12 Krummacher, Rezeptionsgeschichte, S. 224.

[Zurück zum Text]

${ }^{13}$ Goldschmidt, Harry, Um die Sache der Musik, Leipzig: Reclam, 1970, S. 152.

[Zurück zum Text]

14 Noreng, Harald, Der Sprachkünstler und Literaturliebhaber Edvard Grieg, in: Christensen, Mogens e.a. (Hrsg.), Die Gratulanten kommen - Edvard Grieg zum 150. Geburtstag, (Deutsche Hochschulschriften Bd. 814), Egelsbach: Hänsel-Hohenhausen, 1993, S. 77-87.

\section{[Zurück zum Text]}

15 Donnellon, Deirdre, Grieg, Debussy and the Dreyfus Affair, in: British Postgraduate Musicology 1, 1997, S. 14-19.

\section{[Zurück zum Text]}

16 Peters Briefwechsel, S. 319ff. Im Register sind die Nachweise von op. 61 mit denen von op. 60 z. T. vertauscht. In einem Kongreßvortrag aus dem Jahre 1993, veröffentlicht in Studia musicologica norvegica 19, S. 97-102, faßte Hella Brock Griegs skeptische Bemerkungen hingegen folgendermaßen zusammen: "Grieg hat einmal geäußert, er würde sich freuen, wenn diese Lieder auch in Deutschland gesungen würden." (S. 101).

\section{[Zurück zum Text]}

17 Siehe hierzu den Breitkopf Briefwechsel, S. 92, vgl. auch Blok, Vladimir, Grieg - Bartók, Grieg Prokofiev: Aesthetic Parallels, in Studia, 1993, S. 239-250.

\section{[Zurück zum Text]}

${ }^{18}$ Peters Briefwechsel, S. 526.

\section{[Zurück zum Text]}

19 Auch das hat leider eine gewisse Tradition; vgl. Kortsen, Bjarne, Norwegian Music and Musicians, Bergen: Edition Norvegica, ${ }^{2}$ 1977, und seinen Artikel Grieg the Writer - Le style c'est l'homme même, in: Christensen (Hrsg.): Die Gratulanten, S. 58-72. Selbst der jüngst erschienene Band 7 der neuen $M G G$ zeigt in den Artikeln über Norwegen und Oslo u. a. eine einseitige Auswahl an Literatur.

[Zurück zum Text] 
Dokument erstellt am 25. März 1998

PD Dr. Wolfgang Krebs, Clemens Gresser 